\title{
Constructions of Implications Satisfying the Order Property on a Complete Lattice
}

\author{
Yuan Wang ${ }^{1, ~ *, ~ K e m i n g ~ T a n g ~}{ }^{1}$, Zhudeng Wang ${ }^{2}$ \\ ${ }^{1}$ College of Information Science and Technology, Yancheng Teachers University, Yancheng, People's Republic of China \\ ${ }^{2}$ School of Mathematics and Statistics, Yancheng Teachers University, Yancheng, People's Republic of China
}

\section{Email address:}

yctuwangyuan@163.com (Yuan Wang),tkmchina@126.com (Keming Tang), zhudengwang2004@163.com (Zhudeng Wang)

${ }^{*}$ Corresponding author

\section{To cite this article:}

Yuan Wang, Keming Tang, Zhudeng Wang. Constructions of Implications Satisfying the Order Property on a Complete Lattice. Automation, Control and Intelligent Systems. Vol. 5, No. 1, 2017, pp. 1-7. doi: 10.11648/j.acis.20170501.11

Received: January 7, 2017; Accepted: January 19, 2017; Published: February 23, 2017

\begin{abstract}
In this paper, we further investigate the constructions of fuzzy connectives on a complete lattice. We firstly illustrate the concepts of left (right) semi-uninorms and implications satisfying the order property by means of some examples. Then we give out the formulas for calculating the upper and lower approximation implications, which satisfy the order property, of a binary operation.
\end{abstract}

Keywords: Fuzzy Logic, Fuzzy Connective, Implication, Order Property

\section{Introduction}

In fuzzy logic systems (see [1-2]), connectives “and", “or" and "not" are usually modeled by $t$-norms, $t$-conorms, and strong negations on $[0,1]$ (see [3]), respectively. Based on these logical operators on $[0,1]$, the three fundamental classes of fuzzy implications on $[0,1]$, i.e., $R$-, $S$-, and $Q L$-implications on $[0,1]$, were defined and extensively studied (see [4-8]). But, as was pointed out by Fodor and Keresztfalvi [9], sometimes there is no need of the commutativity or associativity for the connectives "and" and "or". Thus, many authors investigated implications based on some other operators like weak $t$-norms [10], pseudo $t$-norms [11], pseudo-uninorms [12], left and right uninorms [13], semi-uninorms [14], aggregation operators [15] and so on.

Uninorms, introduced by Yager and Rybalov [16] and studied by Fodor et al. [17], are special aggregation operators that have proven useful in many fields like fuzzy logic, expert systems, neural networks, aggregation, and fuzzy system modeling. This kind of operation is an important generalization of both $t$-norms and $t$-conorms and a special combination of $t$-norms and $t$-conorms [17]. However, there are real-life situations when truth functions cannot be associative or commutative. By throwing away the commutativity from the axioms of uninorms, Mas et al. introduced the concepts of left and right uninorms on $[0,1]$ in [18] and later in a finite chain in [19], Wang and Fang [13, 20] studied the left and right uninorms on a complete lattice. By removing the associativity and commutativity from the axioms of uninorms, Liu [14] introduced the concept of semi-uninorms and $\mathrm{Su}$ et al. [21] discussed the notion of left and right semi-uninorms on a complete lattice. On the other hand, it is well known that a uninorm (semi-uninorm, left and right uninorms) $U$ can be conjunctive or disjunctive whenever $U(0,1)=0$ or 1 , respectively. This fact allows to use uninorms in defining fuzzy implications [13-14, 22-23].

Constructing fuzzy connectives is an interesting topic. Recently, Wang [24] laid bare the formulas for calculating the smallest pseudo- $t$-norm that is stronger than a binary operation and the largest implication that is weaker than a binary operation, Su and Wang [25] investigated the constructions of implications and coimplications on a complete lattice and Wang et al. [26-28] studied the relations among implications, coimplications and left (right) semi-uninorms on a complete lattice. Moreover, Wang et al. [27, 29-30] investigated the constructions of implications and coimplications satisfying the neutrality principle.

In this paper, based on [24-30], we study the constructions 
of implications satisfying the order property on a complete lattice. After recalling some necessary definitions and examples about the left (right) semi-uninorms and implications on a complete lattice in Section 2, we give out the formulas for calculating the upper and lower approximation implications, which satisfy the order property, of a binary operation in Section 3.

The knowledge about lattices required in this paper can be found in [31].

Throughout this paper, unless otherwise stated, $L$ always represents any given complete lattice with maximal element 1 and minimal element $0 ; J$ stands for any index set.

\section{Left (Right) Semi-Uninorms and Implications}

In this section, we recall some necessary definitions and examples about the left (right) semi-uninorms and implications on a complete lattice.

Definition 2.1 (Su et al. [21]). A binary operation $U$ on $L$ is called a left (right) semi-uninorm if it satisfies the following two conditions:

(U1) there exists a left (right) neutral element, i.e., an element $e_{L} \in L \quad\left(e_{R} \in L\right)$ satisfying $U\left(e_{L}, x\right)=x$ $\left(U\left(x, e_{R}\right)=x\right.$ for all $x \in L$,

(U2) $U$ is non-decreasing in each variable.

If a left (right) semi-uninorm $U$ is associative, then $U$ is the left (right) uninorm [13] on $L$.

If a left (right) semi-uninorm $U$ with the left (right) neutral element $e_{L} \in L \quad\left(e_{R} \in L\right)$ has a right (left) neutral element $e_{R} \in L \quad\left(e_{L} \in L\right)$, then $e_{L}=U\left(e_{L}, e_{R}\right)=e_{R}$. Let $e=e_{L}=e_{R}$. Here, $U$ is the semi-uninorm [14].

For any left (right) semi-uninorm $U$ on $L, U$ is said to be left-conjunctive and right-conjunctive if $U(0,1)=0$ and $U(1,0)=0$, respectively. $U$ is said to be conjunctive if both $U(1,0)=0$ and $U(1,0)=0$ since it satisfies the classical boundary conditions of AND.

$U$ is said to be strict left-conjunctive and strict rightconjunctive if $U$ is conjunctive and for any $x \in L, U(x, 1)=0 \Leftrightarrow x=0 \quad$ and $\quad U(1, x)=0 \Leftrightarrow x=0$, respectively.

Definition 2.2 (Wang and Fang [13]). A binary operation $U$ on $L$ is called left (right) arbitrary $\vee$-distributive if

$$
\begin{array}{r}
U\left(\vee_{j \in J} x_{j}, y\right)=\vee_{j \in J} U\left(x_{j}, y\right) \quad \forall x_{j}, y \in L \\
\left(U\left(x, \vee_{j \in J} y_{j}\right)=\vee_{j \in J} U\left(x, y_{j}\right) \quad \forall x, y_{j} \in L\right) ;
\end{array}
$$

left (right) arbitrary $\wedge$-distributive if

$$
U\left(\wedge_{j \in J} x_{j}, y\right)=\wedge_{j \in J} U\left(x_{j}, y\right) \quad \forall x_{j}, y \in L
$$

$$
\left(U\left(x, \wedge_{j \in J} y_{j}\right)=\wedge_{j \in J} U\left(x, y_{j}\right) \quad \forall x, y_{j} \in L\right)
$$

If a binary operation $U$ is left arbitrary $\vee$-distributive $(\wedge-$ distributive) and also right arbitrary $\vee$-distributive $(\wedge$ -distributive), then $U$ is said to be arbitrary $\vee$-distributive $(\wedge$-distributive).

Noting that the least upper bound of the empty set is 0 and the greatest lower bound of the empty set is 1 , we have

$$
\begin{aligned}
& U(0, y)=U\left(\vee_{j \in \Phi} x_{j}, y\right)=\vee_{j \in \Phi} U\left(x_{j}, y\right)=0 \\
& \left(U(x, 0)=U\left(x, \vee_{j \in \Phi} y_{j}\right)=\vee_{j \in \Phi} U\left(x, y_{j}\right)=0\right)
\end{aligned}
$$

for any $x, y \in L$ when $U$ is left (right) arbitrary $\vee$ -distributive,

$$
\begin{array}{r}
U(1, y)=U\left(\wedge_{j \in \Phi} x_{j}, y\right)=\wedge_{j \in \Phi} U\left(x_{j}, y\right)=1 \\
\left(U(x, 1)=U\left(x, \wedge_{j \in \Phi} y_{j}\right)=\wedge_{j \in \Phi} U\left(x, y_{j}\right)=1\right)
\end{array}
$$

for any $x, y \in L$ when $U$ is left (right) arbitrary $\wedge$ -distributive.

For the sake of convenience, we introduce the following symbols:

$U_{s}^{e_{L}}(L)$ : the set of all left semi-uninorms with the left neutral element $e_{L}$ on $L$;

$U_{s}^{e_{R}}(L)$ : the set of all right semi-uninorms with the right neutral element $e_{R}$ on $L$;

$U_{c s}^{s e_{L}}(L)$ : the set of all strict left-conjunctive left semi-uninorms with the left neutral element $e_{L}$ on $L$;

$U_{c s}^{e_{R} s}(L)$ : the set of all strict right-conjunctive right semi-uninorms with the right neutral element $e_{R}$ on $L$;

$U_{\vee c s}^{s e_{L}}(L)$ : the set of all strict left-conjunctive left arbitrary $\checkmark$-distributive left semi-uninorms with the left neutral element $e_{L}$ on $L$;

$U_{c S}^{e_{R} s}(L)$ : the set of all strict right-conjunctive right arbitrary $\checkmark$-distributive right semi-uninorms with the right neutral element $e_{R}$ on $L$.

Example 2.1 (Su et al. [21]). Let $e_{L} \in L$,

$$
U_{s W}^{e_{L}}(x, y)=\left\{\begin{array}{c}
y \text { if } x \geq e_{L}, \\
0 \text { otherwise, }
\end{array} U_{s M}^{e_{L}}(x, y)=\left\{\begin{array}{c}
y \text { if } x \geq e_{L}, \\
1 \text { otherwise }
\end{array}\right.\right.
$$

$$
U_{c s M}^{e_{L}}(x, y)=\left\{\begin{array}{rr}
0 & \text { if } x=0 \text { or } y=0 \\
y & \text { if } 0<x \leq e_{L}, y \neq 0 \\
1 & \text { otherwise }
\end{array}\right.
$$

where $x$ and $y$ are elements of $L$. Then $U_{s W}^{e_{L}}$ and $U_{s M}^{e_{L}}$ are, respectively, the smallest and greatest elements of 
$U_{s}^{e_{L}}(L)$. By Example 2 and Theorem 8 in [26], we see that $U_{c s}^{s e_{L}}(L)$ and $U_{v c s}^{s e_{L}}(L)$ are two join-semilattices with the greatest element $U_{c s M}^{e_{L}}$.

Example 2.2. Let $e_{L} \in L$,

$$
U_{c s W}^{s e_{L}}(x, y)=\left\{\begin{array}{cc}
y & \text { if } x \geq e_{L}, \\
\wedge\{a \in L \mid a \neq 0\} & \text { if } 0<x \text { not } \geq e_{L}, y=1, \\
0 & \text { otherwise. }
\end{array}\right.
$$

When $\quad e_{L} \neq 0 \quad$ and $\wedge\{a \in L \mid a \neq 0\} \neq 0 \quad, \quad$ it is straightforward to verify that $U_{c s W}^{s e_{L}}$ is a strict left-conjunctive left semi-uninorm with the left neutral element $e_{L}$. If $U \in U_{c s}^{s e_{L}}(L)$, then

$$
U(x, y) \geq\left\{\begin{array}{cc}
U\left(e_{L}, y\right)=y & \text { when } x \geq e_{L}, \\
\wedge\{a \in L \mid a \neq 0\} & \text { when } 0<x \text { not } \geq e_{L}, y=1, \\
0 & \text { otherwise, }
\end{array}\right.
$$

i.e., $U \geq U_{c s W}^{s e_{L}}$. Thus, $U_{c s W}^{s e_{L}}$ is the smallest element of $U_{c s}^{s e_{L}}(L)$

Moreover, assume that $\vee\left\{a \in L \mid a\right.$ not $\left.\geq e_{L}\right\}$ not $\geq e_{L}$. For any $x_{j} \in L(j \in J)$, if $\vee_{j \in J} x_{j} \geq e_{L}$, then there exists $j_{0} \in J$ such that $x_{j_{0}} \geq e_{L}$,

$$
\begin{aligned}
& U_{c s W}^{s e_{L}}\left(\vee_{j \in J} x_{j}, y\right)=y=U_{c s W}^{s e_{L}}\left(x_{j_{0}}, y\right) \\
& =\vee_{j \in J} U_{c s W}^{s e_{L}}\left(x_{j}, y\right) \quad \forall y \in L
\end{aligned}
$$

if $0<\vee_{j \in J} x_{j}$ not $\geq e_{L}$, then $x_{j}$ not $\geq e_{L}$ for any $j \in J$ and there exists $j_{0} \in J$ such that $0<x_{j_{0}}$ not $\geq e_{L}$,

$$
\begin{aligned}
& U_{c s W}^{s e_{L}}\left(\vee_{j \in J} x_{j}, 1\right)=\wedge\{a \mid a \neq 0\}=U_{c s W}^{s e_{L}}\left(x_{j_{0}}, 1\right) \\
& =\vee_{j \in J} U_{c s W}^{s e_{L}}\left(x_{j}, 1\right) ; \\
& \quad U_{c s W}^{s e_{L}}\left(\vee_{j \in J} x_{j}, y\right)=0=U_{c s W}^{s e_{L}}\left(x_{j_{0}}, y\right) \\
& \quad=\vee_{j \in J} U_{c s W}^{s e_{L}}\left(x_{j}, y\right) \quad y \neq 1 ;
\end{aligned}
$$

if $\vee_{j \in J} x_{j}=0$, then $x_{j}=0$ for any $j \in J$,

$$
U_{c s W}^{s e_{L}}\left(\vee_{j \in J} x_{j}, y\right)=0=\vee_{j \in J} U_{c s W}^{s e_{L}}\left(x_{j}, y\right) \quad \forall y \in L .
$$

Therefore, $U_{c s W}^{s e_{L}}$ is left arbitrary $\vee$-distributive and the smallest element of $U_{v c s}^{s e_{L}}(L)$.

Example 2.3. Let $e_{R} \in L$,

$$
U_{s W}^{e_{R}}(x, y)=\left\{\begin{array}{l}
x \text { if } y \geq e_{R}, \\
0 \text { otherwise, }
\end{array} \quad U_{s M}^{e_{R}}(x, y)=\left\{\begin{array}{l}
x \text { if } y \geq e_{R}, \\
1 \text { otherwise }
\end{array}\right.\right.
$$

$$
U_{c s M}^{e_{R}}(x, y)=\left\{\begin{array}{rr}
0 & \text { if } x=0 \text { or } y=0 \\
x & \text { if } 0<y \leq e_{R}, x \neq 0 \\
1 & \text { otherwise }
\end{array}\right.
$$

$$
U_{c s W}^{e_{R} S}(x, y)=\left\{\begin{array}{cc}
x & \text { if } y \geq e_{R}, \\
\wedge\{a \in L \mid a \neq 0\} & \text { if } 0<y \text { not } \geq e_{R}, x=1, \\
0 & \text { otherwise. }
\end{array}\right.
$$

where $x$ and $y$ are elements of $L$. By Example 2.6 in [26], we know that $U_{s W}^{e_{R}}$ and $U_{s M}^{e_{R}}$ are, respectively, the smallest and greatest elements of $U_{s}^{e_{R}}(L)$. By Example 3 and Theorem 8 in [26], we see that $U_{c s}^{e_{R} s}(L)$ and $U_{c s v}^{e_{R} s}(L)$ are two join-semilattices with the greatest element $U_{c s M}^{e_{R}}$.

Similarly, When $e_{R} \neq 0$ and $\wedge\{a \in L \mid a \neq 0\} \neq 0, U_{c s W}^{e_{R} s}$ is the smallest element of $U_{c s}^{e_{R} s}(L)$. Moreover, if $\vee\left\{a \in L \mid a \operatorname{not} \geq e_{R}\right\} \operatorname{not} \geq e_{R}$, then $U_{c s W}^{e_{R} s}$ is the smallest element of $U_{c s \vee}^{e_{R} s}(L)$.

Definition 2.3 (Fodor and Roubens [1], Baczynski and Jayaram [4], Bustince et al. [6], De Baets and Fodor [22]). An implication $I$ on $L$ is a hybrid monotonous (with decreasing first and increasing second partial mappings) binary operation that satisfies the corner conditions $I(0,0)=I(1,1)=1$ and $I(1,0)=0$.

An implication $I$ is said to satisfy the order property with respect to $e$ (w.r. t. e, for short) when $x \leq y$ if and only if $I(x, y) \geq e$ for any $x, y \in L$.

Implications are extensions of the Boolean implication $\rightarrow$ ( $P \rightarrow Q$ meaning that $P$ is sufficient for $Q$ ).

Note that for any implication $I$ on $L$, due to the monotonicity, the absorption principle holds, i.e., $I(0, x)=I(x, 1)=1$ for any $x \in L$.

For the sake of convenience, we introduce the following symbols:

$I(L)$ : the set of all implications on $L$;

$I_{\wedge}(L)$ : the set of all right arbitrary $\wedge$-distributive implications on $L$;

$I^{\text {ope }}(L)$ : the set of all implications which satisfy the order property w. r. t. $e$ on $L$;

$I_{\wedge}^{\text {ope }}(L)$ : the set of all right arbitrary $\wedge$-distributive implications which satisfy the order property w.r.t. $e$ on $L$.

Clearly, $I(L), I_{\wedge}(L), I^{\text {ope }}(L)$ and $I_{\wedge}^{\text {ope }}(L)$ are all meet-semilattices. By Example 2.4 in [25], we know that $I_{\wedge}(L)$ is not a join-semilattice.

Definition 2.3. Let $U$ be a binary operation on $L$. Define $I_{U}^{L}, I_{U}^{R} \in L^{L \times L}$ as follows:

$$
\begin{gathered}
I_{U}^{L}(x, y)=\vee\{z \in L \mid U(z, x) \leq y\} \quad \forall x, y \in L, \\
I_{U}^{R}(x, y)=\vee\{z \in L \mid U(x, z) \leq y\} \quad \forall x, y \in L .
\end{gathered}
$$


Here, $I_{U}^{L}$ and $I_{U}^{R}$ are, respectively, called the left and right residuum of the binary operation $U$.

By virtue of Theorems 4.4 and 4.5 in [13], we know that $U$ and $I_{U}^{R}$ satisfy the following right residual principle:

$$
U(x, z) \leq y \Leftrightarrow z \leq I_{U}^{R}(x, y) \quad \forall x, y, z \in L
$$

when a binary operation $U$ is right arbitrary $\vee$-distributive; $U$ and $I_{U}^{L}$ satisfy the following left residual principle:

$$
U(z, x) \leq y \Leftrightarrow z \leq I_{U}^{L}(x, y) \quad \forall x, y, z \in L
$$

when $U$ is left arbitrary $\vee$-distributive.

When $U$ is non-decreasing in each variable, it is easy to see that $I_{U}^{L}$ and $I_{U}^{R}$ are all decreasing in the first variable and increasing in the second one by Definition 2.3.

Example 2.4. For some left and right semi-uninorms in Examples 2.1-2.3, a simple computation shows that

$$
\begin{aligned}
& I_{U_{c s W}^{s e L}}^{L}(x, y)=\left\{\begin{array}{lr}
0 & \text { if } x=1 \text { and } y=0, \\
1 & \text { if } x \leq y, \\
\vee\left\{a \in L \mid a \text { not } \geq e_{L}\right\} & \text { otherwise, }
\end{array}\right. \\
& I_{U_{c S M}^{e_{L}}}^{L}(x, y)=\left\{\begin{array}{rr}
1 \text { if } x=0 \text { or } y=1, \\
e_{L} \text { if } 0<x \leq y<1, \\
0 \quad \text { otherwise, }
\end{array}\right. \\
& I_{U_{c s W}^{e_{P} s}}^{R}(x, y)=\left\{\begin{array}{lr}
0 & \text { if } x=1 \text { and } y=0, \\
1 & \text { if } x \leq y, \\
\vee\left\{a \in L \mid a \text { not } \geq e_{R}\right\} & \text { otherwise, }
\end{array}\right. \\
& I_{U_{c s M}^{R}}^{R}(x, y)=\left\{\begin{array}{c}
1 \text { if } x=0 \text { or } y=1, \\
e_{R} \text { if } 0<x \leq y<1, \\
0 \quad \text { otherwise, }
\end{array}\right.
\end{aligned}
$$

where $x$ and $y$ are elements of $L$. By the virtue of Theorem 8 in [26], we see that $I_{U_{c s M}^{e_{L}}}^{L}$ is the smallest element of both $I^{o p e_{L}}(L)$ and $I_{\wedge}^{o p e_{L}}(L)$.

When $e_{L} \neq 0$ and $\vee\left\{a \in L \mid a\right.$ not $\left.\geq e_{L}\right\}$ not $\geq e_{L}$, it is easy to see that $I_{U_{c s W}^{s e_{L}}}^{L}$ is the greatest element of $I^{o p e_{L}}(L)$.

Moreover, assume that $\wedge\{a \in L \mid a \neq 0\} \neq 0$. For any $y_{j} \in L(j \in J)$, if $\wedge_{j \in J} y_{j}=0$, then there exists $j_{0} \in J$ such that $y_{j_{0}}=0$,

$$
\begin{aligned}
& I_{U_{c s W}^{s e L}}^{L}\left(x, \wedge_{j \in J} y_{j}\right)=I_{U_{c s W}^{s e L}}^{L}(x, 0)=I_{U_{c s w}^{s e L}}^{L}\left(x, y_{j_{0}}\right) \\
& =\wedge_{j \in J} I_{U_{c s w}^{s s . L}}^{L s}\left(x, y_{j}\right) \quad \forall x \in L ;
\end{aligned}
$$

if $x \leq \wedge_{j \in J} y_{j}$, then $x \leq y_{j}$ for any $j \in J$,

$$
I_{U_{c s W}^{s e_{W}}}^{L}\left(x, \wedge_{j \in J} y_{j}\right)=1=\wedge_{j \in J} I_{U_{c s W}^{s e_{L}}}^{L}\left(x, y_{j}\right) \quad \forall x \in L
$$

if $0<\wedge_{j \in J} y_{j}$ not $\geq x$, then $0<y_{j}$ for any $j \in J$ and there exists $j_{0} \in J$ such that $0<y_{j_{0}}$ not $\geq x$,

$$
\begin{gathered}
I_{U_{c s W}^{s e L}}^{L}\left(x, y_{j}\right) \geq \vee\left\{a \in L \mid a \operatorname{not} \geq e_{L}\right\} ; \\
I_{U_{c s W}^{s e L}}^{L}\left(x, \wedge_{j \in J} y_{j}\right)=\vee\left\{a \in L \mid a \operatorname{not} \geq e_{L}\right\} \\
=I_{U_{c s W}^{s e L}}^{L}\left(x, y_{j_{0}}\right)=\wedge_{j \in J} I_{U_{c s W}^{s e L}}^{L}\left(x, y_{j}\right) .
\end{gathered}
$$

Therefore, $I_{U_{c s W}^{s e W_{W}}}^{L}$ is the greatest element of $I_{\wedge}^{\text {ope }_{L}}(L)$.

$I_{U_{c s M}^{e_{L}}}^{L} \quad$ Similar conclusions hold for $I^{o p e_{R}}(L)$ and $I_{\wedge}^{o p e_{R}}(L)$.

\section{Constructing the Implications Satisfying the Order Property}

Recently, Su and Wang [25] have studied the constructions of implications and coimplications and Wang et al. [27, 29-30] further investigated the constructions of implications and coimplications satisfying the neutrality principle on a complete lattice.

This section is a continuation of $[25,27,29-30]$. We will study the constructions of the upper and lower approximation implications which satisfy the order property.

It is easy to verify that if $J \neq \Phi$, then

$$
I_{j} \in I^{o p e_{L}}(L) \forall j \in J \Rightarrow \wedge_{j \in J} I_{j} \in I^{o p e_{L}}(L) .
$$

When $e_{L} \neq 0$ and $\vee\left\{a \in L \mid a\right.$ not $\left.\geq e_{L}\right\}$ not $\geq e_{L}$, we see that $I^{o p e_{L}}(L)$ is also a complete lattice with the smallest element and greatest element $I_{U_{c s W}^{s e L}}^{L}$ by Example 2.4. Thus, for a binary operation $A$ on $L$, if there exists $I \in I^{o p e_{L}}(L)$ such that $A \leq I$, then

$$
\wedge\left\{I \mid A \leq I, I \in I^{o p e_{L}}(L)\right\}
$$

is the smallest implication that is stronger than $A$ and satisfies the order property w. r. t. $e_{L}$ on $L$. Here, we call it the upper approximation implication, which satisfies the order property w. r. t. $e_{L}$, of $A$ and write as $[A)_{I}^{o p e_{L}}$. Similarly, if there exists $I \in I^{o p e_{L}}(L)$ such that $I \leq A$, then

$$
\vee\left\{I \mid I \leq A, I \in I^{o p e_{L}}(L)\right\}
$$

is the largest implication that is weaker than $A$ and satisfies the order property w. r. t. $e_{L}$ on $L$. Here, we call it the lower approximation implication, which satisfies the order property w. r. t. $e_{L}$, of $A$ and write as $(A]_{I}^{o p e_{L}}$.

Likewise, for a binary operation $A$ on $L$, we may introduce the following symbols: 
$[A)_{I}^{o p e_{R}}$ : the upper approximation implication, which satisfies the order property w. r. t. $e_{R}$, of $A$;

$(A]_{I}^{\text {ope } e_{R}}$ : the lower approximation implication, which satisfies the order property w. r. t. $e_{R}$, of $A$;

$[A)_{I}^{o e_{L} \wedge}\left([A)_{I}^{o p e_{R} \wedge}\right)$ : the upper approximation right arbitrary $\wedge$-distributive implication, which satisfies the order property w. r. t. $e_{L}\left(e_{R}\right)$, of $A$;

$(A]_{I}^{o e_{L} \wedge}\left((A]_{I}^{o p e_{R} \wedge}\right)$ : the lower approximation right arbitrary $\wedge$-distributive implication, which satisfies the order property w. r. t. $e_{L}\left(e_{R}\right)$, of $A$.

Definition 3.1 (see Su and Wang [25]). Let $A$ be a binary operation on $L$. Define the upper approximation implicator $A_{u i}$ and the lower approximation implicator $A_{l i}$ of $A$ as follows:

$$
\begin{array}{ll}
A_{u i}(x, y)=\vee\{A(u, v) \mid u \geq x, v \leq y\} & \forall x, y \in L, \\
A_{l i}(x, y)=\wedge\{A(u, v) \mid u \leq x, v \geq y\} & \forall x, y \in L .
\end{array}
$$

Theorem 3.1 (see Su and Wang [25]). Let $A, B \in L^{L \times L}$. Then the following statements hold:

$$
A_{l i} \leq A \leq A_{u i} .
$$

$$
(A \vee B)_{u i}=A_{u i} \vee B_{u i} \text { and }
$$

$$
(A \wedge B)_{l i}=A_{l i} \wedge B_{l i} .
$$

$A_{u i}$ and $A_{l i}$ are hybrid monotonous.

If $A$ is are hybrid monotonous, then $A_{u i}=A_{l i}=A$.

Theorem 3.2. Let $A \in L^{L \times L}$.

(1) If $A$ is right arbitrary $\vee$-distributive, then $A_{u i}$ is also right arbitrary $\vee$-distributive,

$$
\begin{gathered}
\left(I_{A}^{R}\right)_{l i}=I_{A_{u a}}^{R},\left(I_{A}^{R}\right)_{u i} \leq I_{A_{l a}}^{R}, \\
A_{u a}\left(x,\left(I_{A}^{R}\right)_{l i}(x, y)\right) \leq y \quad \forall x, y \in L .
\end{gathered}
$$

(2) If $A$ is right arbitrary $\wedge$-distributive, then $A_{l i}$ is also right arbitrary $\wedge$-distributive.

(3) If $A$ is left arbitrary $\vee$-distributive, then,

$$
\begin{gathered}
\left(I_{A}^{L}\right)_{l i}=I_{A_{u l a}}^{L},\left(I_{A}^{L}\right)_{u i} \leq I_{A_{l a}}^{L}, \\
A_{u a}\left(\left(I_{A}^{L}\right)_{l i}(x, y), x\right) \leq y \quad \forall x, y \in L .
\end{gathered}
$$

Proof. We only prove that statement (1) holds.

Assume that $A$ is a right arbitrary $\vee$-distributive binary operation on $L$. Clearly, $A_{u a}$ is also right arbitrary $\vee$ -distributive. By Definition 3.1, the monotonicity of $A$ and
$I_{A}^{R}$, and the right residual principle, we have that

$$
\begin{gathered}
I_{A_{u u}}^{R}(x, y)=\vee\left\{z \in L \mid A_{u a}(x, z) \leq y\right\} \\
=\vee\{z \in L \mid \vee\{A(u, v) \mid u \leq x, v \leq z\} \leq y\} \\
=\vee\{z \in L \mid \vee\{A(u, z) \mid u \leq x\} \leq y\} \\
=\vee\{z \in L \mid A(u, z) \leq y \forall u \leq x\} \\
=\vee\left\{z \in L \mid z \leq I_{A}^{R}(u, y) \forall u \leq x\right\} \\
=\vee\left\{z \in L \mid z \leq \wedge_{u \leq x} I_{A}^{R}(u, y)\right\} \\
=\wedge_{u \leq x} I_{A}^{R}(u, y) \forall x, y \in L, \\
\left(I_{A}^{R}\right)_{l i}(x, y)=\wedge\left\{I_{A}^{R}(u, v) \mid u \leq x, v \geq y\right\} \\
=\wedge\left\{I_{A}^{R}(u, y) \mid u \leq x\right\}=\wedge_{u \leq x} I_{A}^{R}(u, y) \forall x, y \in L .
\end{gathered}
$$

Thus, $\left(I_{A}^{R}\right)_{l i}=I_{A_{u a}}^{R}$. Similarly, we have that

$$
\begin{gathered}
\left(I_{A}^{R}\right)_{u i}(x, y)=\vee\left\{I_{A}^{R}(u, y) \mid u \geq x\right\} \forall x, y \in L, \\
A_{l a}(x, z)=\wedge\left\{A\left(u_{1}, v\right) \mid u_{1} \geq x, v \geq z\right\} \\
=\wedge\left\{A\left(u_{1}, v\right) \mid u_{1} \geq x\right\} \forall x, z \in L, \\
\left(I_{A_{a l}}^{R}\right)(x, y) \\
=\vee\left\{z \in L \mid \wedge\left\{A\left(u_{1}, z\right) \mid u_{1} \geq x\right\} \leq y\right\} \forall x, y \in L .
\end{gathered}
$$

If $u \geq x$, let $z=I_{A}^{R}(u, y)$, then

$$
\begin{aligned}
& A(u, z)=A(u, \vee\{c \in L \mid A(u, c) \leq y\}) \\
& =\vee\{A(u, c) \mid A(u, c) \leq y\} \leq y, \\
& \wedge\left\{A\left(u_{1}, z\right) \mid u_{1} \geq x\right\} \leq A(u, z) \leq y .
\end{aligned}
$$

So, $\left(I_{A}^{R}\right)_{u i}(x, y) \leq\left(I_{A_{l i}}^{R}\right)(x, y)$ for any $x, y \in L$, i.e., $\left(I_{A}^{R}\right)_{u i} \leq I_{A_{l a}}^{R}$.

Moreover, we know that $A_{u a}$ is right arbitrary $\vee$ -distributive and hence

$$
\begin{aligned}
& A_{u a}\left(x,\left(I_{A}^{R}\right)_{l i}(x, y)\right)=A_{u a}\left(x, I_{A_{u a}}^{R}(x, y)\right) \\
& =A_{u a}\left(x, \vee\left\{z \in L \mid A_{u a}(x, z) \leq y\right\}\right) \\
& =\vee\left\{A_{u a}(x, z) \mid A_{u a}(x, z) \leq y\right\} \leq y \forall x, y \in L .
\end{aligned}
$$

The theorem is proved.

Below, we give out the formulas for calculating the upper and lower approximation implications which satisfy the order property.

Theorem 3.3. Suppose that $A \in L^{L \times L}, e_{L} \neq 0$ and $\vee\left\{a \in L \mid a\right.$ not $\left.\geq e_{L}\right\}$ not $\geq e_{L}$.

(1) If $A \leq I_{U_{\text {ssid }}^{s e l}}^{L}$, then $[A)_{I}^{o p e_{L}}=I_{U_{\text {csi }}^{c}}^{L} \vee A_{u i}$;

$$
\text { if } A \geq I_{U_{c s M}^{e_{c}}}^{L} \text {, then }(A]_{I}^{o p e_{L}}=I_{U_{c s W}^{s e L}}^{L} \wedge A_{l i} \text {. }
$$

(2) If $\wedge\{a \in L \mid a \neq 0\} \neq 0, A \geq I_{U_{\text {cLs }}^{e^{L}}}^{L}$ and $A$ is right 
arbitrary $\wedge$-distributive, then

$$
(A]_{I}^{o p e_{L} \wedge}=I_{U_{c s W}^{s e L}}^{L} \wedge A_{l i} .
$$

Moreover, if $A$ is non-decreasing in its first variable, then $(A]_{I}^{o p e_{L} \wedge}=I_{U_{c s W}^{s e L}}^{L} \wedge A$.

Proof. Assume that $\vee\left\{a \in L \mid a\right.$ not $\left.\geq e_{L}\right\}$ not $\geq e_{L}$ and $e_{L} \neq 0$. Then $I_{U_{c s M}^{e_{L}}}^{L}$ and $I_{U_{c s W}^{s e_{L}}}^{L}$ are, respectively, the smallest and greatest elements of $I^{o p e_{L}}(L)$ by Example 2.4.

(1) If $A \leq I_{U_{c s W}^{s e_{L}}}^{L}$, let $I_{1}=I_{U_{c s M}^{e_{L}}}^{L} \vee A_{u i}$, then $A \leq I_{1}$ and

$$
I_{U_{c s M}^{e_{L}}}^{L} \leq I_{1} \leq I_{U_{c s W}^{s e L}}^{L}
$$

Thus, $I_{1}(0,0)=I_{1}(1,1)=1$ and $I_{1}(1,0)=0$. If $x \leq y$, then $I_{1}(x, y) \geq I_{U_{c s M}^{e_{L}}}^{L}(x, y) \geq e_{L} \quad ; \quad$ if $\quad I_{1}(x, y) \geq e_{L} \quad$, then $I_{U_{c s W}^{s e_{L}}}^{L}(x, y) \geq I_{1}(x, y) \geq e_{L}$ and so $x \leq y$, i.e., $I_{1}$ satisfies the order property w. r. t. $e_{L}$. By Theorem 3.1 (3) and the hybrid monotonicity of $I_{U_{c s M}^{e_{L}}}^{L}$, we know that $I_{1}$ is hybrid monotonous. So, $I_{1} \in I^{o p e_{L}}(L)$. If $A \leq I$ and $I \in I^{o p e_{L}}(L)$, then $A_{u i} \leq I_{u i}=I$ and $I_{1}=I_{U_{c s M}^{s e L}}^{L} \vee A_{u i} \leq I$. Therefore,

$$
[A)_{I}^{o p e_{L}}=I_{U_{c s M}^{s e_{L}}}^{L} \vee A_{u i} .
$$

If $A \geq I_{U_{c s M}^{e L}}^{L}$, let $I_{2}=I_{U_{c s W}^{s e L}}^{L} \wedge A_{l i}$, then $I_{2} \leq A$,

$$
A_{l i} \geq\left(I_{U_{c s M}^{e_{L}}}^{L}\right)_{l i}=I_{U_{c s M}^{e_{L}}}^{L}, I_{U_{c s M}^{e_{L}}}^{L} \leq I_{2} \leq I_{U_{c s W}^{s e L}}^{L}
$$

Thus, we can prove in an analogous way that $I_{2} \in I^{\text {ope }_{L}}(L)$ and $(A]_{I}^{o p e_{L}}=I_{U_{c s W}^{s e_{W}}}^{L} \wedge A_{l i}$.

(2) When $\wedge\{a \in L \mid a \neq 0\} \neq 0, \quad I_{U_{c s M}^{e_{L}}}^{L}$ and $I_{U_{c s}^{s e L}}^{L} \quad$ are, respectively, the smallest and greatest elements of $I_{\wedge}{ }^{o p e_{L}}(L)$ by Example 2.4. Let $I_{3}=I_{U_{c s W}^{s e V}}^{L} \wedge A_{l i}$. If $A \geq I_{U_{c s M}^{e_{L}}}^{L}$, then $I_{3} \in I^{\text {ope }_{L}}(L)$ by statement (1). Noting that $A$ is right arbitrary $\wedge$-distributive, we can see that $A_{l i}$ is also right arbitrary $\wedge$-distributive by Theorem 3.2 (1). So, $I_{3}$ is right arbitrary $\wedge$-distributive, i.e., $I_{3} \in I_{\wedge}{ }^{o p e_{L}}(L)$. By the proof of statement (1), we know that $(A]_{I}^{o p e_{L} \wedge}=I_{U_{c s W}^{s e_{L}}}^{L} \wedge A$.

Moreover, if $A$ is non-decreasing in its first variable, then $A_{l i}=A$ by Theorem 3.1 (4) and so

$$
(A]_{I}^{o p e_{L} \wedge}=I_{U_{c s W}^{s e e_{L}}}^{L} \wedge A .
$$

The theorem is proved.

Analogous to Theorem 3.3, we have the following theorem.
Theorem 3.4. Suppose that $A \in L^{L \times L}, e_{R} \neq 0$ and $\vee\left\{a \in L \mid a\right.$ not $\left.\geq e_{R}\right\}$ not $\geq e_{R}$.

(1) If $A \leq I_{U_{c s W}^{e_{R S}}}^{R}$, then $[A)_{I}^{\text {ope } e_{R}}=I_{U_{c s M}^{e_{R}}}^{R} \vee A_{u i}$;

$$
\text { if } A \geq I_{U_{c s M}^{e_{R}}}^{R} \text {, then }(A]_{I}^{\text {ope }} e_{R}=I_{U_{c s W}^{e_{R} s}}^{R} \wedge A_{l i} .
$$

(2) If $\wedge\{a \in L \mid a \neq 0\} \neq 0, \quad A \geq I_{U_{c s M}^{e_{R}^{R}}}^{e^{e_{R}}}$ and $A$ is right arbitrary $\wedge$-distributive, then

$$
(A]_{I}^{\text {ope } e_{R} \wedge}=I_{U_{c s W}^{e R^{s}}}^{R} \wedge A_{l i} .
$$

Moreover, if $A$ is non-decreasing in its first variable, then $(A]_{I}^{\text {ope }_{R} \wedge}=I_{U_{c s}^{e_{R} S}}^{R} \wedge A$.

\section{Conclusions and Future Works}

Constructing fuzzy connectives is an interesting topic. Recently, Wang et al. [24-25, 27, 29-30] investigated the constructions of implications and coimplications on a complete lattice. In this paper, motivated by these works, we give out the formulas for calculating the upper and lower approximation implications, which satisfy the order property, of a binary operation.

In a forthcoming paper, we will investigate the relationships between left (right) semi-uninorms and implications on a complete lattice.

\section{Acknowledgements}

This work is supported by Science Foundation of Yancheng Teachers University (16YCKLQ006), the National Natural Science Foundation of China (61379064) and Jiangsu Provincial Natural Science Foundation of China (BK20161313).

\section{References}

[1] J. Fodor and M. Roubens, "Fuzzy Preference Modelling and Multicriteria Decision Support", Theory and Decision Library, Series D: System Theory, Knowledge Engineering and Problem Solving, Kluwer Academic Publishers, Dordrecht, 1994.

[2] G. J. Klir and B. Yuan, "Fuzzy Sets and Fuzzy Logic, Theory and Applications", Prentice Hall, New Jersey, 1995.

[3] E. P. Klement, R. Mesiar and E. Pap, "Triangular Norms", Trends in Logic-Studia Logica Library, Vol. 8, Kluwer Academic Publishers, Dordrecht, 2000.

[4] M. Baczynski and B. Jayaram, "Fuzzy Implication”, Studies in Fuzziness and Soft Computing, Vol. 231, Springer, Berlin, 2008.

[5] M. Baczynski and B. Jayaram, " $Q L$-implications: some properties and intersections", Fuzzy Sets and Systems, 161, 158-188, 2010. 
[6] H. Bustince, P. Burillo and F. Soria, "Automorphisms, negations and implication operators", Fuzzy Sets and Systems, 134, 209-229, 2003.

[7] F. Durante, E. P. Klement, R. Mesiar and C. Sempi, "Conjunctors and their residual implicators: characterizations and construction methods", Mediterranean Journal of Mathematics, 4, 343-356, 2007.

[8] Y. Shi, B. Van Gasse, D. Ruan and E. E. Kerre, "On dependencies and independencies of fuzzy implication axioms", Fuzzy Sets and Systems, 161, 1388-1405, 2010.

[9] J. Fodor and T. Keresztfalvi, "Nonstandard conjunctions and implications in fuzzy logic", International Journal of Approximate Reasoning, 12, 69-84, 1995.

[10] J. Fodor, "Srict preference relations based on weak $t$-norms", Fuzzy Sets and Systems, 43, 327-336, 1991.

[11] Z. D. Wang and Y. D. Yu, "Pseudo-t-norms and implication operators on a complete Brouwerian lattice", Fuzzy Sets and Systems, 132, 113-124, 2002.

[12] Y. Su and Z. D. Wang, "Pseudo-uninorms and coimplications on a complete lattice", Fuzzy Sets and Systems, 224, 53-62, 2013.

[13] Z. D. Wang and J. X. Fang, "Residual operators of left and right uninorms on a complete lattice", Fuzzy Sets and Systems, 160, 22-31, 2009.

[14] H. W. Liu, "Semi-uninorm and implications on a complete lattice", Fuzzy Sets and Systems, 191, 72-82, 2012.

[15] Y. Ouyang, "On fuzzy implications determined by aggregation operators", Information Sciences, 193, 153-162, 2012.

[16] R. R. Yager and A. Rybalov, "Uninorm aggregation operators", Fuzzy Sets and Systems, 80, 111-120, 1996.

[17] J. Fodor, R. R. Yager and A. Rybalov, "Structure of uninorms", Internat. J. Uncertainly, Fuzziness and Knowledge-Based Systems, 5, 411-427, 1997.

[18] M. Mas, M. Monserrat and J. Torrens, "On left and right uninorms", Internat. J. Uncertainly, Fuzziness and Knowledge-Based Systems, 9, 491-507, 2001.

[19] M. Mas, M. Monserrat and J. Torrens, "On left and right uninorms on a finite chain", Fuzzy Sets and Systems, 146, 3-17, 2004.

[20] Z. D. Wang and J. X. Fang, "Residual coimplicators of left and right uninorms on a complete lattice", Fuzzy Sets and Systems, 160, 2086-2096, 2009.

[21] Y. Su, Z. D. Wang and K. M. Tang, "Left and right semi-uninorms on a complete lattice", Kybernetika, 49, 948-961, 2013.

[22] B. De Baets and J. Fodor, "Residual operators of uninorms", Soft Computing, 3, 89-100, 1999.

[23] M. Mas, M. Monserrat and J. Torrens, "Two types of implications derived from uninorms", Fuzzy Sets and Systems, 158, 2612-2626, 2007.

[24] Z. D. Wang, "Generating pseudo-t-norms and implication operators", Fuzzy Sets and Systems, 157, 398-410, 2006.

[25] Y. Su and Z. D. Wang, "Constructing implications and coimplications on a complete lattice", Fuzzy Sets and Systems, 247, 68-80, 2014.

[26] X. Y. Hao, M. X. Niu and Z. D. Wang, "The relations between implications and left (right) semi-uninorms on a complete lattice", Internat. J. Uncertainly, Fuzziness and Knowledge-Based Systems, 23, 245-261, 2015.

[27] X. Y. Hao, M. X. Niu, Y. Wang and Z. D. Wang, "Constructing conjunctive left (right) semi-uninorms and implications satisfying the neutrality principle", Journal of Intelligent and Fuzzy Systems, 31, 1819-1829, 2016.

[28] M. X. Niu, X. Y. Hao and Z. D. Wang, "Relations among implications, coimplications and left (right) semi-uninorms", Journal of Intelligent and Fuzzy Systems, 29, 927-938, 2015.

[29] Z. D. Wang, M. X. Niu and X. Y. Hao, "Constructions of coimplications and left (right) semi-uninorms on a complete lattice", Information Sciences, 317, 181-195, 2015.

[30] Z. D. Wang, "Left (right) semi-uninorms and coimplications on a complete lattice", Fuzzy Sets and Systems, 287, 227-239, 2016.

[31] G. Birkhoff, "Lattice Theory", American Mathematical Society Colloquium Publishers, Providence, 1967. 